\title{
Focus. Érudition, voyage et écriture : les notes de lecture de Gérard de Nerval sur l'Égypte
}

\section{Rémy Arcemisbéhère}

\section{(2) OpenEdition}

1 Journals

\section{Édition électronique}

URL : https://journals.openedition.org/genesis/5692

DOI : 10.4000/genesis.5692

ISSN : 2268-1590

Éditeur :

Presses universitaires de Paris Sorbonne (PUPS), Société internationale de génétique artistique littéraire et scientifique (SIGALES)

Édition imprimée

Date de publication : 15 décembre 2020

Pagination : 101-106

ISBN : 979-10-231-0704-3

ISSN : 1167-5101

\section{Référence électronique}

Rémy Arcemisbéhère, «Focus. Érudition, voyage et écriture : les notes de lecture de Gérard de Nerval sur l'Égypte », Genesis [En ligne], 51 | 2020, mis en ligne le 20 décembre 2021, consulté le 07 février 2022. URL : http://journals.openedition.org/genesis/5692 ; DOI : https://doi.org/10.4000/genesis. 5692 


\title{
Érudition, voyage et écriture : les notes de lecture de Gérard de Nerval sur l'Égypte
}

\author{
Rémy Arcemisbéhère
}

L 'œuvre de Gérard de Nerval met régulièrement en scène la lecture : au fil de ses textes, l'écrivain se peint en lecteur et signale sa culture livresque par d'abondantes pratiques intertextuelles (référence, citation, réécriture, plagiat) ${ }^{1}$. En accordant la préséance à la lecture sur l'écriture dans ses textes, l'écrivain consacre l'hégémonie d'une bibliothèque dont il affirme qu'elle «a de quoi rendre fou un sage ${ }^{2}$. Dans les faits, Nerval fut surtout un lecteur dilettante pour qui la quantité et la diversité des ouvrages fréquentés importait davantage que la qualité de la lecture : au sein des nombreuses listes bibliographiques qu'il déploie dans ses œuvres, des ouvrages lus en entier en côtoient d'autres connus uniquement par des fragments, par des recensions ou par leur seul titre. Le caractère superficiel de ces lectures informe en retour l'esthétique : Nerval met en œuvre un récit excentrique, vif et allègre, empruntant son paradigme à la promenade. Le mouvement d'élaboration de ses œuvres témoigne d'une volonté d'atteindre un point d'équilibre où la lecture, la vie et l'écriture cessent d'être en concurrence : ses notes préparatoires constituent donc une négociation; elles rendent compte d'un parcours de la bibliothèque dont la fonction est moins de nourrir le récit que de conquérir une légitimité 3 . La rédaction du Voyage en Orient est exemplaire de ce processus exogénétique : de nombreuses lectures préparent et accompagnent l'expérience du voyage ainsi que sa mise en récit ${ }^{4}$. Trois documents liés à sa genèse et se situant précisément à l'interface entre lecture et écriture illustrent ce travail nervalien des textes.

\section{Une fiche de lecture}

Avant et après son voyage en Égypte de 1843, Nerval réalisa de nombreuses recherches sur l'Égypte : la bibliothèque de $l^{\prime}$ Institut conserve une fiche de lecture 5 sur laquelle il a compulsé la matière de plusieurs notices de la Bibliothèque orientale d'Herbelot de Molainville concernant les religions ${ }^{6}$. Ces notes peuvent avoir préparé ou suivi le voyage de 1843 : dans les deux cas, elles ont servi à l'élaboration du Voyage en Orient ${ }^{7}$. L'organisation de ce document manuscrit où Nerval recense la matière de quatre notices de la Bibliothèque orientale est peu orthodoxe : ses notes commencent en haut à gauche de la feuille et se poursuivent de manière circulaire autour de la page, de sorte que le centre de la page est laissé vierge. Nerval circule donc de notice en notice grâce au système de renvoi et tente d'organiser sur l'espace de la page un vaste système dont

1. Jean-Pierre Richard a affirmé que l'œuvre de Nerval «ne possède aucun droit de propriété» (Poésie et profondeur, Paris, Seuil, 1927, p. 75).

2. Aurélia, $N P l$ III, p. 743. Ce même jugement négatif à l'égard des livres, qualifiés de «nourriture malsaine pour l'âme», apparaît dans la préface des Illuminés ( $N P l$ II, p. 887). Les citations de Nerval sont extraites de l'édition de ses œuvres dans la Pléiade sous la direction de Jean Guillaume et de Claude Pichois (Paris, Gallimard, t. I, 1989, t. II, 1984, t. III, 1993), noté $N P l$ suivi du tome concerné.

3. À partir de son premier internement en maison de santé en 1841, Nerval traverse une crise de réputation et ambitionne dès lors de fournir au public parisien les garanties de son retour à la raison.

4. Publié en 1851 , cet ouvrage reprend la matière de feuilletons parus entre 1838 et 1850 et condense la matière de plusieurs voyages faits en Europe, en Égypte, au Liban et en Turquie.

5. D. 741 f $^{\circ} 67$, Bibliothèque de l'Institut, Fonds Spoelberch de Lovenjoul. 6. Barthélémy d'Herbelot de Molainville, Bibliothèque orientale ou Dictionnaire universel [...], Paris, Compagnie des Libraires, 1697, 3 volumes. Cette somme érudite constitua pour lui une source de matériaux, d'images, de noms et de connaissances tout au long de sa vie : Théophile Gautier affirme que Nerval lut et prit des notes sur «toute la bibliothèque orientale» dans le cadre d'un projet, jamais réalisé, d'opéra intitulé La Reine de Saba (Théophile Gautier, Portraits littéraires, Paris, Michel Lévy, 1875, p. 18-19).

7. Le conte maçonnique «Histoire de la reine du Matin et de Soliman» inséré dans le récit de voyage ainsi que dans plusieurs articles de la même période s'appuient sur ces connaissances. 
les fondements lui échappent encore. Cela explique aussi le choix de se tourner vers un ouvrage qui, préfigurant le travail des mythologues, met en regard différents systèmes religieux $^{8}:$ la lecture s'assimile donc ici à une quête d'un principe secret d'unité9.

Sa prise de notes opère un véritable travail de sélection et de composition : il élude une partie des notices, relève les figures secondaires au détriment des principales et articule les données ainsi prélevées à d'autres lectures ${ }^{10}$. Ce document illustre le goût de Nerval pour la constitution de systèmes mais aussi pour le composite : à partir des notices, il élabore une chimère textuelle, un ensemble issu du mélange d'éléments hétéroclites. Les notes de Nerval font pour cela la part belle aux figures de monstres et aux récits de gigantomachies : il relève par exemple une phrase entière relative au palais du géant Argenk et à sa «galerie, dans laquelle étaient peints les portraits de toutes les créatures raisonnables qui avaient habité la terre avant la création d'Adam »11. Comme cette galerie de créatures antédiluviennes issues d'accouplements monstrueux, les notes de Nerval amalgament des discours anciens sur la mythologie. Ce travail d'hybridation initié par la lecture sera poursuivi dans l'écriture : ainsi, le recueil Les Chimères met pareillement en œuvre un «art prométhéen qui se revendique comme une contre-création 12 ».

\section{Un journal de lecture}

Si une partie de ces notes sera reprise de manière diffuse dans les textes de Nerval, celles prises lors de son séjour cairote en 1843 constituent le matériau génétique exclusif du Voyage en Orient. Ce voyage fut polarisé par la nécessité d'écrire : Nerval affirme en effet être venu au Caire «pour travailler, pour étudier la ville»13. Il occupa une grande partie de son temps dans les rayons de la bibliothèque de la Société égyptienne qui possédait un fonds spécialisé sur l'Égypte issu de dons de savants ${ }^{14}$. Il ambitionne de mener en parallèle le voyage dans le monde et le parcours de la bibliothèque et écrit à son père le 14 février : «Nous avons [à la Société égyptienne] tous les livres possibles concernant l'Égypte, ce qui me permet d'étudier, à mesure que je vois les choses ${ }^{15}$. » Pourtant, cette équivalence entre lecture et voyage ne se maintiendra pas : le projet de visiter la Haute-Égypte qu'il souhaitait réaliser après avoir «épuisé la bibliothèque égyptienne ${ }^{16}$ » finit par avorter. Les livres prennent alors le pas sur l'expérience du voyage; ils ne la complètent plus mais se substituent à elle.

Le carnet de notes qu'il rédigea lors de son séjour cairote 17 rend compte de cette métamorphose du rapport entre voyage, érudition et écriture. Réorganisé tardivement 18 , cet ensemble incomplet est composé de deux parties : la première (feuillets 1 à 16) contient les impressions de voyage et les notes de lecture de l'auteur, la seconde (feuillets 17 à 25 ) est réservée aux comptes de ses frais de voyage, qu'il a tenus du mardi 7 février

8. Voir François Pouillon (dir.), Dictionnaire des orientalistes de langue française, Paris, Karthala, 2008, p. 896.

9. Par exemple, la recension de la notice «Caïn» élude les passages relatifs à la Genèse et insiste sur les éléments du mythe retenus par les traditions orientales qui réhabilitent la figure du fratricide, révélant l'envers de ce récit et en même temps, sa cohérence.

10. Dans la notice consacrée à la montagne «Càf», seuls sont retenus les éléments les plus fabuleux (pourtant raillés par l'auteur de la Bibliothèque orientale), et il associe la montagne Càf à l'anneau atlas alors que ce détail n'est pas mentionné par d'Herbelot. Dans la notice consacrée à Soliman Ben Daoud, il concentre son attention sur la figure peu connue de Thamurath.

11. Barthélémy d'Herbelot de Molainville, Bibliothèque orientale [...], op. cit., I, p. 455 (souligné par Nerval).

12. Bertrand Marchal, «Les chimères de Nerval», Nerval. Actes du colloque de la Sorbonne du 15 novembre 1997, Paris, Presses de 1'Université Paris-Sorbonne, 1997, p. 123.

13. Voyage en Orient, NPl II, p. 315.

14. Voir Jean-Marie Carré, Voyageurs et écrivains en Égypte, Genève, Stalkine Reprints, 2006, p. 27. Gérard de Nerval ainsi qu'un grand nombre de critiques confondent la Société égyptienne et l'Association littéraire de l'Égypte car les deux associations partageaient les mêmes locaux (sur cette distinction, voir Auriant, «Les origines de l'Institut égyptien. La société égyptienne (1836-1859)», Journal des savants, no 5, 1926, p. 227).

15. Lettre à son père du 14 février 1843, NPl I, p. 1391

16. Lettre à son père du 18 mars $1843, N P l \mathrm{I}, \mathrm{p} .1394$.

17. NAF 14282, Bibliothèque nationale de France, Cabinet des manuscrits. Nous appuyons sur la transcription réalisée par Pierre Martino ( «L carnet du Voyage en Orient de Gérard de Nerval », Revue de littérature comparée, no 3, 1933, p. 140-173).

18. L'ordre initial peut être reconstitué en s'appuyant sur la taille et la forme d'une tache d'huile présente sur la plupart des pages : les feuillets 1 à 3 qui correspondent bien au début du carnet étaient suivis par les feuillets 6 à 10 puis par les feuillets 11 à 16 et enfin par les feuillets 4 et 5 . 
au samedi 1er avril 1843. Dans la première section, les premiers feuillets, constitués de dessins et d'impressions de voyage, ne contiennent que très peu d'indications bibliographiques ${ }^{19}$, des notes de lecture plus longues apparaissent à partir du sixième feuillet : elles sont donc postérieures au 8 février 1843, date à laquelle Nerval régla son inscription à la bibliothèque de l'Association littéraire d'Égypte ${ }^{20}$. L'utilisation d'encres différentes au sein d'une même page témoigne du fait que chaque section de notes engagea plusieurs sessions de travail en bibliothèque. Enfin, à partir du verso du septième feuillet, les citations se font plus nombreuses et plus longues. Des impressions de voyage et des notes touristiques s'intègrent ensuite à cette prise de notes jusqu'au quatorzième feuillet. On identifie donc au maximum deux séries de prises de notes en bibliothèque.

Deux tendances semblent avoir animé la production de ce carnet : le voyageur tente d'appliquer le programme qu'il s'est fixé en relevant d'abord les références de livres à lire, puis en recopiant de longs passages de ces ouvrages savants sur l'histoire, la géographie et la religion égyptienne. Pourtant, cette posture ne sera pas maintenue : selon Camille Aubaude, ces notes témoignent surtout d'un «éclectisme» et d'une «absence de plan de travail $21 »$. S'il recopie consciencieusement de longs passages de la chronologie de l'Histoire mahométane (qu'il ne reprendra dans aucune de ses œuvres), la plupart de ses recensions se détache du propos et de la structure de l'ouvrage original pour n'en retenir que des détails (couleurs, noms, anecdotes). Tout en mimant le sérieux de l'étude, l'écrivain recueille donc au gré du hasard et avec une certaine désinvolture des fragments de textes pour peupler son récit à venir selon une démarche analogue à celle qui préside à la collecte d'impressions de voyage. En outre, la description matérielle du Carnet $d u$ Caire démontre que, lors de son séjour, Nerval associait ces deux domaines : ses notes de lecture sont encadrées par des souvenirs personnels et par un registre de comptes. L'organisation du carnet nuance le primat des lectures sur l'expérience : les lectures apparaissent fragmentées, réparties en petites sessions, consacrées à des objets divers. Ces notes mettent en lumière un processus de création lui aussi mixte, associant l'activité du lecteur à l'expérience du voyageur et faisant collaborer le pôle du vécu et celui de la lecture.

\section{Des annotations}

Un autre document permet de préciser la nature de l'opération exogénétique réalisée par Nerval en Égypte : il s'agit d'un exemplaire de L'Égypte de Murtadi conservé à la bibliothèque de l'Institut français d'archéologie orientale du Caire et où, sur la deuxième de couverture, une série de références manuscrites correspondent à l'écriture de Nerval ${ }^{22}$. Ce document se présente comme une liste de vingt items composés chacun d'un intitulé suivi d'une référence paginée. L'ouvrage annoté constitue une source importante du Voyage en Orient : Nerval en réécrit plusieurs passages dans les chapitres «L'Île de Roddah» et «Les Pyramides»23. Néanmoins, L'Égypte de Murtadi n'apparaît pas dans le Carnet du Caire; en outre, les annotations sur ce livre ne concernent pas les cent premières pages du Murtadi qui ont inspiré le Voyage en Orient. Il faut donc faire l'hypothèse que d'autres notes existent ou que ces passages correspondent aux pages manquantes du Carnet $d u$ Caire. Les références sur le livre constitueraient alors une sorte de programme des passages que l'auteur se préparait à recopier plus tard, ce qui démontrerait que le lecteur de 1843 procédait activement au recueil du matériau de son récit de voyage en bibliothèque.

19. À l'exception de celles réalisées à partir de périodiques probablement lus dans un cabinet de lecture : dans le Voyage en Orient, il affirme avoir souscrit à un abonnement au cabinet de Madame Bonhomme.

20. Dans son carnet de compte, au recto du 17 e feuillet, à la fin de la colonne des comptes notée « 8 ?» (8 février 1843) apparaît la mention «Bibliothèque» suivi de la somme « 1 p. 20 ».

21. Camille Aubaude, Le Voyage en Égypte de Gérard de Nerval, Paris, Kimé, 1998, p. 56.

22. L'Égypte de Murtadi, fils du Gaphiphe, ou il est traité des Pyramides, $d u$ débordement $d u \mathrm{Nil}, \&$ des autres merveilles de cette Province, selon les opinions \& traditions des Arabes..., trad. Pierre Vattier Paris, Lovys Billaine, 1666 (conservé à l'Institut français d'archéologie orientale sous la cote Res. Prec. A3). L'histoire de ce fonds est complexe : il semble qu'après la dissolution de la société savante, les ouvrages qui la composaient furent acquis par Auguste Mariette, puis à la mort de ce dernier, par le gouvernement français afin de servir de noyau à la bibliothèque de la mission archéologique du Caire qui deviendra l'Institut français d'archéologie orientale. La graphie des lettres et des chiffres ainsi que la présentation sérielle très similaire à la seconde partie du Carnet $d u$ Caire conduisent à identifier Gérard de Nerval comme l'auteur de ces notes.

23. Voir la table des concordances réalisée par Jean Richer dans Nerval et les doctrines ésotériques (Paris, Griffon d'or, 1947, p. 34-38 et p. 48-53). 


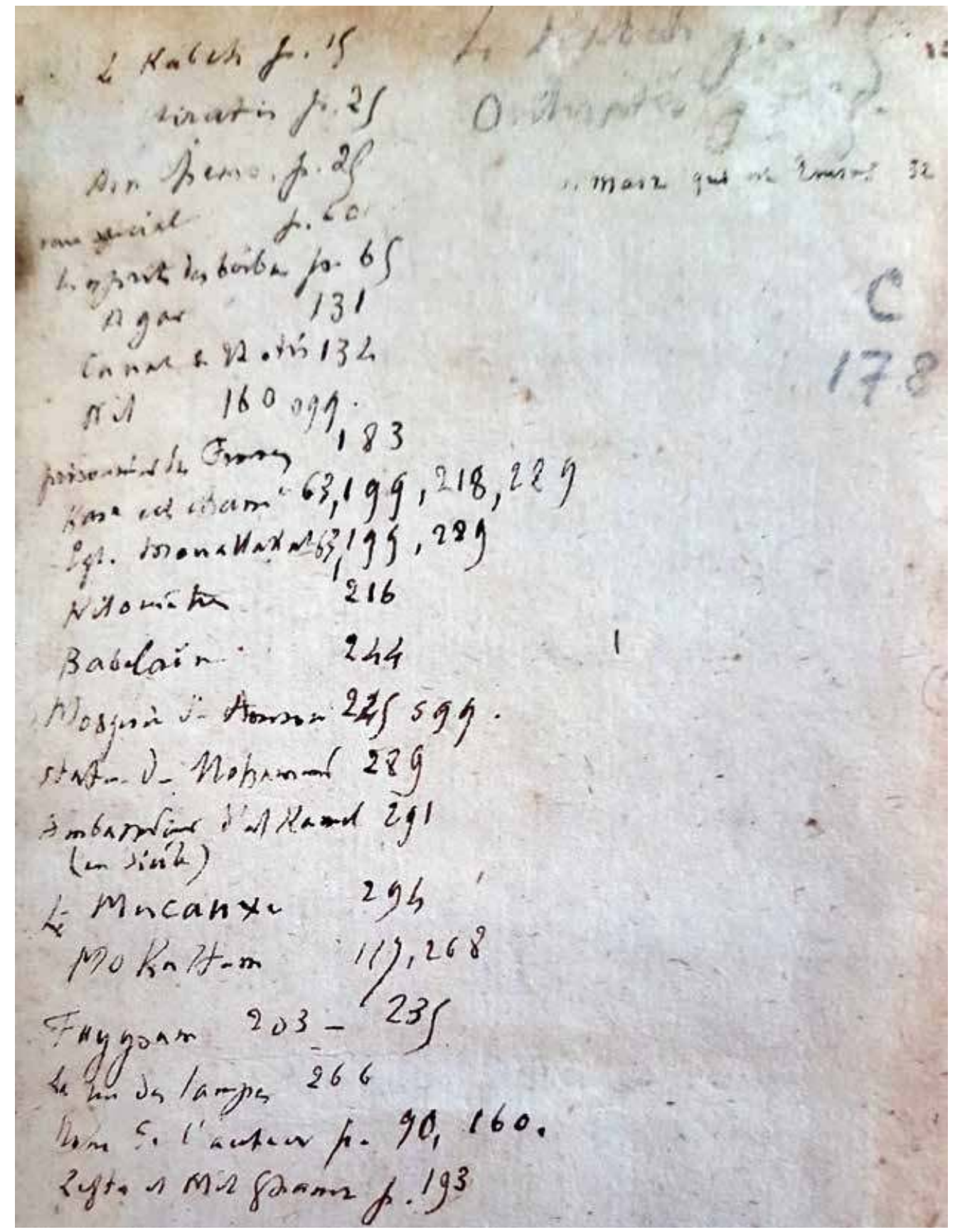

Fig. 1 : Notes de Nerval prises sur la deuxième de couverture de L'Égypte de Murtadi, fils du Gaphiphe [...], trad. Pierre Vattier, Paris, Lovys Billaine, 1666. 
Qu'elles préparent ou prolongent le travail effectué dans le Carnet du Caire, ces notes participent du même projet : par la forme du sommaire, le lecteur sélectionne et met à disposition de l'écrivain une matière narrative, la réduisant à sa forme la plus elliptique pour permettre sa transformation. Si quelques items de la liste concernent des textes que Nerval réutilisera directement ${ }^{24}$, les autres occurrences renvoient à des sujets divers (récits fabuleux ou bibliques, description de lieux imaginaires ou réels, anecdotes historiques) et s'articulent pour la plupart à des thématiques importantes de l'imaginaire nervalien : Nerval relève systématiquement les figures féminines évoquées par Vattier, il souligne plusieurs épisodes d'enfermement et se montre attentif aux manifestations de la magie en Égypte et au thème de l'homme face au surnaturel, amalgamant ainsi ses préoccupations au contenu du livre de Vattier ${ }^{25}$. Par cette lecture, il entend également retrouver «la ville des Mille et Une Nuits ${ }^{26}$ » que le réel lui a confisquée : ces annotations constituent une tentative pour compenser le processus de désenchantement et témoignent d'une progressive retraite dans la bibliothèque.

Cette liste fait également apparaître une préférence pour les récits des origines et les récits alternatifs aux grands textes religieux : le choix de recenser cet ouvrage s'explique donc par le caractère original et originel octroyé par Nerval à ces sources arabes. L'antériorité du savoir présenté dans le Murtadi est soulignée dans la préface de l'ouvrage où Vattier rappelle que les Arabes «ont inventé les Sciences occultes [...], ont élevé les hauts bâtiments et [...] ont gravé leurs sciences sur les pierres molles ${ }^{27} \gg$. Ainsi, le parcours de ce livre a d'abord vocation à amorcer l'écriture du voyage en lui octroyant une caution : l'écrivain tente de retrouver dans ces sources une authenticité que la bibliothèque $\mathrm{a}$ confisqué à l'expérience du voyageur.
Edward Saïd affirme que «la pure et simple transcription des textes érudits étaient les seuls vaisseaux possibles» pour Nerval en Orient ${ }^{28}$. L'examen génétique de ces trois documents permet de nuancer ce constat polarisé par la lecture des dernières œuvres de Nerval. Le voyage de 1843 a joué un rôle critique dans l'évolution du rapport de l'auteur à la bibliothèque : d'abord points de repère symbolique permettant l'accès au réel, les sources livresques ont progressivement recouvert celui-ci. Les notes de Nerval sont le théâtre de cette concurrence entre le voyage dans le monde et celui qui a lieu dans la bibliothèque : par leur forme à la fois fragmentaire et mixte, elles tentent de réduire l'empire des lectures et de ménager un espace de création entre les textes et en leur sein même, décomposant la matière lue pour l'ouvrir à toutes les recompositions. Cette crise est le véritable sujet du Voyage en Orient : cette œuvre hybride qui associe impressions de voyage, digressions livresques et même plagiats est moins le récit d'un voyage informé par les livres que l'histoire de la confrontation d'un lecteur au monde. La conscience de cet écart ne cessera par la suite de se renforcer pour Nerval : le narrateur d'Isis craint ainsi «en voyage, de gâter par des lectures faites d'avance, l'impression première des lieux célèbres 29 ».

24. La mention «Moquée d'Amrou» identifie un passage utilisé dans l'élaboration du chapitre III des «Femmes du Caire» (Voyage en Orient, NPl II, p. 344).

25. L'épisode de la statue du Prophète prise pour le Prophète lui-même par des Andalous se détache en particulier, car il met en scène le thème de la duplicité qui est structurant dans le Voyage en Orient.

26. Voyage en Orient, NPl II, p. 262.

27. L'Égypte de Murtadi, op. cit., p. 5.

28. Edward Saïd, L'Orientalisme. L'Orient créé par l'Occident, Paris, Seuil, 1982, p. 212.

29. Les Filles du feu, NPl III, p. 617. 
RÉMY ARCEMISBÉHÈRE est doctorant à la faculté des Lettres de Sorbonne Université ; il réalise une thèse sur le travail des sources dans l'œuvre de Gérard de Nerval sous la direction Sophie Basch. Il est affilié au Cellf (Centre d'étude de la langue et de la littérature française), unité mixte du CNRS et de Sorbonne Université. Il participe au projet «Bibliothèques d'Orient » coordonné par la BnF.

remy.arcemisbehere@gmail.com

\section{Érudition, voyage et écriture : les notes de lecture de Gérard de Nerval sur l'Égypte}

Cette étude interroge les rapports entre exogenèse et intertextualité à partir du cas représentatif de Gérard de Nerval. La rédaction du Voyage en Orient manifeste de façon exemplaire le processus exogénétique : de nombreuses lectures préparent et accompagnent l'expérience du voyage ainsi que de sa mise en récit. Trois documents manuscrits associés à la genèse du Voyage en Orient permettent de mieux cerner l'élaboration de l'œuvre à partir des lectures de l'écrivain, ce travail des sources orientales : une fiche de lecture réalisée à la Bibliothèque royale compulsant plusieurs notices de la Bibliothèque orientale d'Herbelot de Molainville au sujet des religions orientales, le carnet qui accompagna l'écrivain lors de son séjour cairote en 1843 et les notes réalisées par l'auteur directement sur un ouvrage, L'Égypte du Murtadi, qu'il consulta en Égypte.

The aim of this study is to illustrate the relationships between exogenesis and intertextuality in Gérard de Nerval's Voyage en Orient. The writing of Voyage en Orient shows the exogenetic process in an exemplary way. Nerval read actively in preparation for the journey, during the journey, and during the writing process. Three handwritten documents associated with his activities as a reader - this work of the Orientalist sourcing of Voyage en Orient allow us to better understand the development of the work : reading notes on several entries from Herbelot de Molainville's Bibliothèque orientale, which he consulted at the Bibliothèque Royale; the poet's personal notebook from his Cairo trip in 1843; and his handwritten annotations on L'Égypte du Murtadi, which he gained access to in Egypt.

Die vorliegende Arbeit hinterfragt die Beziehung zwischen Exogenese und Intertextualität anhand des repräsentativen Falles von Gérard de Nerval. Die Niederschrift der Voyage en Orient ist eine beispielhafte Manifestation des exogenetischen Prozesses : Zahlreiche Lesungen bereiten die Erfahrung der Reise und ihre Erzählung vor und begleiten sie. Drei handschriftliche Dokumente, die mit der Entstehung der Voyage en Orient in Verbindung stehen, ermöglichen es uns, die Entwicklung des Werkes auf der Grundlage der Lektüren des Schriftstellers, diese eigentliche Arbeit an den orientalischen Quellen, besser zu verstehen : eine in der Königlichen Bibliothek hergestellte Lesekarte, die mehrere Notizen aus der Bibliothèque orientale über orientalische Religionen von Herbelot de Molainville zusammenstellt, das Notizbuch, das den Schriftsteller während seines Aufenthalts in Kairo 1843 begleitete, und die Notizen, die der Autor direkt zu einem Werk, L'Égypte $d u$ Murtadi, gemacht hat, das er in Ägypten konsultierte.
Este artículo trata de las relaciones entre exogénesis e intertextualidad a partir de un caso representativo de Gerard de Nerval. La redacción de Voyage en Orient pone de manifiesto de manera ejemplar el proceso exogenético: numerosas lecturas preparan y acompañan la experiencia del viaje así como la ejecución de su relato. Tres documentos manuscritos asociados a la génesis de Voyage en Orient permiten entender mejor la elaboración de la obra a partir de las lecturas del escritor, esa reelaboración de las fuentes orientales: una ficha de lectura realizada en la Biblioteca Real en la que coteja varios datos de la Bibliothèque orientale de Herbelot de Molainville acerca de las religiones orientales, el carnet que acompaña al escritor durante su estadía en El Cairo en 1843 y las notas inscritas por el autor directamente en un libro, L'Égypte $d u$ Murtadi, que consulta en Egipto.

Este estudo questiona as relações entre exogénese e intertextualidade a partir do caso representativo de Gérard de Nerval. A redação de Voyage en Orient evidencia de forma exemplar o processo exogenético: numerosas leituras preparam e acompanham a experiência da viagem, bem como a sua narrativa. Três documentos manuscritos associados à génese de Voyage en Orient permitem compreender melhor a elaboração da obra a partir das leituras do escritor, um trabalho sobre fontes orientais: uma ficha das leituras realizadas na Biblioteca Real sobre vários artigos da Bibliothèque orientale de Herbelot de Molainville sobre as religiões orientais, o caderno que acompanhou o escritor durante a sua estadia no Cairo em 1843 e as notas feitas pelo autor diretamente numa obra, L'Égypte du Murtadi, que consultou no Egito.

L'articolo interroga i rapporti tra esogenesi e intertestualità in Gérard de Nerval. La redazione del Viaggio in Oriente manifesta in modo esemplare il processo esogenetico: numerose letture preparano e accompagnano l'esperienza del viaggio e la sua narrazione. Tre documenti manoscritti, associati alla genesi del Viaggio in Oriente, permettono d'inquadrare meglio l'elaborazione dell'opera a partire dalle letture dello scrittore: una scheda di lettura realizzata alla Bibliothèque royale, durante la consultazione di numerose voci della Bibliothèque orientale di Herbelot de Molainville sulle religioni orientali, il carnet che ha accompagnato lo scrittore nel suo soggiorno cairota del 1843 e le note realizzate dall'autore direttamente su un testo, L'Égypte du Murtadi, consultato in Egitto. 